\title{
The comparison between curcumin and propolis against sepsis-induced oxidative stress, inflammation, and apoptosis in kidney of adult male rat
}

\author{
Areeg Abd-Elrazek ${ }^{1 *}$ D, Sahar Mahmoud ${ }^{2}$ and Ahmed Abd ElMoneim ${ }^{3}$
}

\begin{abstract}
Background: Propolis is a honeybee product displaying an anti-inflammatory, antimicrobial, and antioxidant effect on several tested animal models. Curcumin a polyphenol extracted from turmeric that gained interest as a potentially safe and inexpensive treatment for kidney diseases.

The present study aimed to compare the protective effects of curcumin and propolis on endotoxemia-induced renal dysfunction.

Results: Sepsis induction caused a marked decline in renal GSH, GPx, and GR, as well as antioxidant enzyme activities; CAT and SOD. Elevation in LPO, NO, IL-1 $\beta$, and PGE2 contents were observed as well. A marked induction in Bax contents, Bax \Bcl2 ratio, accompanied by activation of NF-kB in the kidney of sepsis-induced rats was reported. However, Prop pretreatment of endotoxemic rats was effective in controlling the depletion of renal GSH content and its correlated enzymes; Cur was more potent in maintaining the renal CAT and SOD contents, as well as, dimensioning LPO content. Despite the renal inflammatory marker IL-1 $\beta, P G E 2, N O$ contents, $B a x \backslash B C l_{2}$ ratio, and NF-kB activation were greatly reduced by both curcumin and propolis, only Cur pretreatment attenuated NF-kB activation in kidney tissue of septic rat.

Conclusion: Though pretreatment of either Cur or Prop to septic rats protected their kidneys against oxidation, inflammation, and apoptosis status, Cur pretreatment was superior in protecting rats' kidney after sepsis induction.
\end{abstract}

Keywords: Nephrotoxicity, Endotoxemia, Anti-inflammatory markers, Anti-apoptotic markers, Oxidative stress

\section{Background}

Despite the advances in medical care, sepsis still become the major cause of complex clinical morbidity in intensive care units [1]. In patients, the response progresses from inflammation to septic shock and multiple organ dysfunction syndrome leading to death. Therefore, sepsis is considered a complex systemic illness representing the deregulated host response to infection [2]. Research on sepsis including studies on pathogens, coagulation,

\footnotetext{
*Correspondence: areeg.abdelrazek@gmail.com

${ }^{1}$ Researcher at Department of Physiology, National Organization of Drug Control, and Research, Cairo, Egypt

Full list of author information is available at the end of the article
}

immune responses, organ damage, sepsis, and septic shock have revealed sepsis as an important risk factor for acute renal failure evidenced by the sudden decline in kidney function [1].

Previous experimental data have shown that inflammatory mediators may influence abnormalities in renal tubular cells so renal damage occurs at sepsis attack. The recommendations of these studies guided scientists to investigate a promising concept concerning the pathogenesis of acute renal damage in sepsis [3]. The associations between inflammatory factors and kidney oxygenation, metabolism, inflammation, and function, where these factors promote leukocyte aggregation, which may result in 
renal inflammation [4]. On the other hand, apoptosis is believed to have a key role in sepsis kidney failure. Overexpression of $\mathrm{Bcl}-2$ prevented apoptotic cell death of lymphocytes during sepsis [3]. Apoptosis may be initiated by many factors, including circulating glucocorticoid contents, pro-inflammatory cytokines such as TNF-a, interleukin-1, reactive oxygen radicals, and nitric oxide.

Furthermore, changes in inflammatory cytokines (TNF- $\alpha$, and IL- $1 \alpha$ ) that are the crucial factors leading to acute renal failure. Interleukin-1 (IL-1 $\alpha)$ is one of several proinflammatory cytokines produced during infection, sepsis, and the systemic inflammatory response syndrome. Excessive IL-1 release has been directly conducted to the progression of hypotension, multi-organ dysfunction, septic shock, and death in patients and animals with sepsis.

Prostaglandin E (PGE) pharmacologically affects vasodilation and platelet aggregation to protect vascular endothelium and improve microcirculation. PGE can regulate endothelial cells and macrophages to produce anti-inflammatory and cell protection functions.

During sepsis, activation of inhibitor of nuclear factor kappa-B (NF- $\mathrm{kB}$ ) and its consequences occurs among all organs that eventually lead to organ failure accompanied by increased morbidity and mortality. Therefore, NF- $\mathrm{KB}$ became an attractive target for therapy and pharmacological control of endotoxemia [5].

Natural products are used as remedies, all over the world, for several medical conditions in humans. They are not only effective but also safe in therapeutic doses below their toxic values [6]. Since inflammatory responses are a critical factor during sepsis progression, the clearance of inflammatory mediators in vivo is an important direction for the prevention and treatment of septic acute renal failure [4].

Sepsis may be induced by several models, and the most common model is cecal ligation and puncture. This model results in three insults to the host: (1) surgical trauma to the tissues, (2) ischemic tissue from the ligated cecum, and (3) polymicrobial sepsis from fecal spillage after needle puncture(s) [7].

The second model lipopolysaccharides (LPS) injection is a simple and sterile way to induce sepsis, and it shares many similarities with the initial phase of human sepsis. However, LPS-induced sepsis models show weakness in preclinical research; furthermore, it is expensive as compared to other models [8].

On the other hand, the cecal slurry model of sepsis is based upon intra-peritoneal injection of cecal contents from a donor rodent that have been standardized in quantity (i.e., fecal mass) and suspended in fluid. The obvious advantage of this technique between cecal slurry and the two models is that rats injected with cecal slurry is easy, lack the surgical tissue trauma and ischemic tissue generated by standard CLP methods, rather than it is cheap as compared to LPS [7].

Curcumin (Cur) is a principal constituent found in turmeric, which is used as an edible component regarding to its flavor, color, and medicinal properties. Curcumin was used in treating a variety of health problems, including respiratory illness, liver disorders, inflammatory disorders, and diabetic wounds [9]. It was reported to have anticarcinogenic, hepatoprotective, cardioprotective, and thrombosuppresive effects [10].

Propolis (Prop) is a resin enriched with saliva and enzymatic secretions of bees collected from plants. Bees use Prop to cover hive walls, fill gaps, and embalm killed invader insects [11]. Propolis is a natural product that gained attention as folk medicine due to its chemical composition as it contains a variety of flavonoids, phenols, alcohols, terpenes, sterols, vitamins, and amino acids [12]. Several studies demonstrated the antibacterial, antifungal, anti-inflammatory, antioxidant, immunomodulatory, and wound healing properties of propolis extract [13]. Developing sepsis therapy requires a deep understanding of the pathogenesis of sepsis and organ dysfunction. Although investigations indicated the renoprotective effect of propolis [14], and curcumin [15], there is no time to investigate more efficacious treatments due to critical nature of sepsis. The present study was designed to investigate the effect of cecal slurry as animal model of sepsis on antioxidant parameters, antiinflammatory, and apoptotic markers that indicate the sepsis infection. On the other hand, this study established to evaluate and compare between the protective effect (curcumin or propolis) against inflammation, oxidative stress, and apoptosis induced by cecal slurry as animal model of sepsis.

\section{Methods}

All animal experimentation protocols were carried out under the supervision of the Ethical Committee of NODCAR (NODCAR/II/21/19), after approving of general assembly of biological control and research (No. 234\2019).

Seventy healthy (6-8 weeks old) adult male Wister albino rats brought from animal house of NODCAR, weighing between 150 and $200 \mathrm{~g}$, were kept under strict hygienic conditions for acclimatization under standard condition ( $12 \mathrm{~h} \mathrm{light/dark}$ cycle, 20 to $22{ }^{\circ} \mathrm{C}$ temperature) with food and water ad libitum.

\section{Preparation of cecal slurry}

Sepsis was induced using a modified cecal slurry model [16]. Donor rats were anesthetized with an intramuscular injection of ketamine hydrochloride $(50 \mathrm{mg} / \mathrm{kg})$ and xylazine $(10 \mathrm{mg} / \mathrm{kg})$. A midline laparotomy was made, and the cecum was expelled. A $0.5 \mathrm{~cm}$ cut was 
performed on the other side of the mesenteric surface of the cecum, and the cecum was pressed to expel feces. The feces were assembled and weighed, then were diluted 1:3 with a $5 \%$ dextrose solution immediately. The cecal slurry from several donor rats was homogenate then shaking to create a uniform suspension before treatment. The new cecal slurry was used within $2 \mathrm{~h}$ of preparation [16].

\section{Induction of sepsis}

The cecal slurry from donor rats was freshly prepared, diluted, and then injected intraperitoneally to rats to induced sepsis [16].

\section{Propolis}

Propolis was purchased from College of Agriculture, Cairo University, grounded and extracted by $95 \%$ ethanol, for 1 week at room temperature and kept away from light. The ethanolic solution was filtered to eliminate wax and solid particles, ethanol was evaporated to obtain propolis extract which was then dissolved in $0.5 \%$ carboxyl methyl cellulose (CMC) just before administration as $250 \mathrm{mg} / \mathrm{kg} /$ day [17].

\section{Curcumin}

Curcumin powder (curcuminoid; 95.02\%) was obtained from Sigma chemical company (Sigma-Aldrich, USA). Curcumin powder was suspended in $0.5 \%$ CMC just before administration at dose $(200 \mathrm{mg} / \mathrm{kg} /$ day $)$ [9].

\section{Experimental design and animal grouping}

The experimental animals used in this study (60 adult male albino rats; each weighing 150-200 g) were equally divided into six groups. Sample size calculated by Gpower software. The groups were as follows: (I) control group $(\mathrm{CON})$ : animals received $0.5 \% \mathrm{CMC}(0.5 \mathrm{ml} / \mathrm{rat})$ every day by oral gavage for 14 days and followed by $5 \%$ D5W IP on the 15th day. (II) Sepsis group (SEP), animals received an oral administration of $0.5 \%$ CMC daily $(0.5 \mathrm{ml} / \mathrm{rat})$ for 14 days. Then, sepsis was induced experimentally on the $15^{\text {th }}$ day by intraperitoneal injection of freshly prepared cecal slurry. (III) Propolis group (Prop), animals were orally administered Prop in a dose of $250 \mathrm{mg} / \mathrm{kg} /$ days $(0.5 \mathrm{ml} / \mathrm{rat})$ for 14 days followed by D5W on the 15th day. (IV) Curcumin group (Cur), animals of this group were received curcumin orally in a dose of $250 \mathrm{mg} / \mathrm{kg} /$ days $(0.5 \mathrm{ml} / \mathrm{rat})$ for 14 days then received D5W on the 15th day. (V) Propolis and sepsis group (Prop and SEP), animals were orally administrated propolis $(250 \mathrm{mg} / \mathrm{kg} /$ day $)(0.5 \mathrm{ml} / \mathrm{rat})$ for 14 days, on the 15th day injected IP by cecal slurry at $200 \mathrm{mg} / \mathrm{kg}$ bw. (VI) Curcumin and sepsis group (Cur and SEP), rats of this group have orally administrated the curcumin (250 $\mathrm{mg} / \mathrm{kg} /$ days) $(0.5 \mathrm{ml} / \mathrm{rat})$ for 14 days then on the 15 th day injected IP by cecal slurry. The total volume of cecal slurry and intraperitoneal D5W was $10 \mathrm{ml} / \mathrm{kg}$.

Animals of all groups were anesthetized with ketamine hydrochloride $(100 \mathrm{mg} / \mathrm{kg} \mathrm{bw})$, and sacrificed on the 16th day post-treatment, kidneys were excised, one kidney was quickly removed on an ice plate, washed with saline, and all kidney samples from all groups were kept at $-80{ }^{\circ} \mathrm{C}$ for further investigations. The other kidney from rats of all treated groups were washed with saline, then plotted and preserved directly in formalin for histological investigations.

\section{Tissues samples preparation}

Rat kidney tissues from all treated groups were homogenized in iced phosphate buffer $(0.05 \mathrm{M}, \mathrm{pH} 7.4)$, centrifuged at $3500 \mathrm{rpm}$ for $5 \mathrm{~min}$. Supernatants of all kidney tissue samples were obtained and used for estimation of oxidant/antioxidant markers of oxidative stress, Il- $1 \beta$, and PGE-2 contents.

\section{Determination of oxidative stress markers (LPO, GSH, GPx, GR, and NO) and antioxidant enzyme activities (CAT and SOD) in kidney homogenate by spectrophotometer} Lipid peroxidation assay (LPO); Nitric oxide (NO); the content of reduced glutathione were determined by using commercial kits from Biodiagnostics, Egypt, according to the manufacturers' protocols.

The activities of glutathione peroxidase (GPx); glutathione reductase (GR); superoxide dismutase (SOD), and catalase (CAT) activities were measured according to commercial kits from Biodiagnostics, Egypt, according to the manufacturers' protocols.

Determination of inflammatory markers (IL-1 $\beta$, PGE2, Bcl2 , and $B a x$ ) in kidney homogenate of adult male rat by ELISA

Kidney tissues homogenate were used to determine the pro-inflammatory cytokines, tumor necrosis factor- $\alpha$ (TNF- $\alpha)$, interleukin-1 $\beta$ (IL-1 $\beta)$, the inflammatory mediator, prostaglandin E2 (PGE2), and apoptosis biomarkers (Bcl-2 and Bax) contents. These parameters were estimated using commercially available ELISA (enzymelinked immunosorbent assay) kits according to the manufacturers' protocols.

\section{Histopathological examination}

Kidney tissue samples were fixed in 10\% neutralbuffered formalin, dehydrated, impeded in paraffin wax, and sectioned $(5 \mu \mathrm{m})$. They were stained further with hematoxylin and eosin according to the method described by Bancroft and Cook [18] for light microscopy investigation (Nikon Eclipse E200-LED, Tokyo, Japan). 


\section{Immunohistochemical analysis}

For determination of NF-k $\beta$ expression, kidney samples were prepared as sections ( $5 \mu \mathrm{m}$ thickness) and blocked with $0.1 \%$ of $\mathrm{H}_{2} \mathrm{O}_{2}$ containing methanol for $15 \mathrm{~min}$ to block the endogenous peroxidase. Sections were incubated with a rabbit polyclonal NF-k $\beta$ antibody at $4{ }^{\circ} \mathrm{C}$ overnight. Thereafter, the sections were washed with PBS (phosphate-buffered saline) and incubated with biotinylated goat anti-rabbit immunoglobulins followed by streptavidin-peroxidase complexes at $30{ }^{\circ} \mathrm{C}$ for $30 \mathrm{~min}$. The peroxidase activity was developed using diaminobenzidine (DAB)-hydrogen peroxide [19]. Images were captured with an original magnification of $\times 400$ (Nikon Eclipse E200-LED, Tokyo, Japan).

\section{Statistical studies}

Data are expressed as means \pm standard error (SEM) and examined by one-way ANOVA using a statistical package program (SPSS version 20.0). The difference between groups was performed by using Tukey's multiple comparison test.

\section{Results}

The present study showed that cecal slurry injection caused a reduction in the of GSH content, GPx, GR significantly at $P<0.05$ (Fig. 1), CAT, and SOD activities (Fig. 2) while causing a significant $(P<0.05)$ increase in oxidative stress markers (LPO and NO) (Fig. 2) in kidney tissue homogenate in sepsis group. Meanwhile, the present results recorded a significant elevation $(P<0.05)$ in inflammatory markers (IL-1 $\beta$, TNF- $\alpha$, and PGE2) in the kidney tissues of the sepsis group (Fig. 4). Sepsis also caused a significant $(P<0.05)$ rise in Bax content accompanied to a significant depletion $(P<0.05)$ in $\mathrm{Bcl}-2$ content which are the key proteins of apoptosis.

On the other hand, pretreatment with either propolis or curcumin caused a significant $(P<0.05)$ increase in kidney GSH content, GPx, and GR activity and prevent its depletion concerning values of SEP-treated rat group (Fig. 1). As depicted from Fig. 2, both propolis and curcumin enhanced kidney SOD and CAT activities with a significant change at $(P<0.05)$ as compared to sepsis non-treated group. Meanwhile, propolis increased CAT and SOD activities significantly $(P<0.05)$ as compared to sepsis rat group as shown in (Fig. 2). Both propolis and curcumin administration prevented the elevation of the renal LPO content with a non-significant change of propolis and curcumin-treated rats groups, respectively. Propolis and curcumin treatments were found to prevent the sharp increase recorded in kidney LPO content at $P$ $<0.05$ if compared with sepsis-induced rat group, although a significant change at $P<0.05$ still recorded when compared to the values of the control rats group (Fig. 2). The data in Fig. 2 reported that both treatments suppress the increase of renal $\mathrm{NO}$ contents significant at $P<0.05$ as compared to septic-rat group, though still recording an increase significantly $(P<0.05)$ as compared to control rats.

Meanwhile, the curcumin and propolis preadministration inhibited the increased contents of inflammatory markers (IL-1 $\beta$, TNF- $\alpha$, and PGE2) in the kidney significantly as compared to SEP-rat group, although the effect of curcumin is slightly better (Fig. 3).

Both propolis and curcumin pretreatment deceased significantly $(P<0.05)$ the Bax content, in contrast, elevated $\mathrm{Bcl}-2$ contents in kidney tissues as compared to SEP-rat group. The Bax/Bcl2 ratio of propolis and curcumin revealed a significant decrease with respect to sepsis control group (Fig. 4); the effect of curcumin is more pronounced.

\section{Histopathological examination}

Histological investigation of rat kidneys from control group revealed the normal structure of kidney indicated by normal glomeruli with intact Bowman's capsule and proximal convoluted tubules. Kidneys of sepsis-induced rats showed dilation of the interstitial tubule, severe tubular leakage, atrophic glomeruli, and interstitial hemorrhages. Kidneys of septic rats pretreated with curcumin indicated an improvement compared with a kidney of sepsis-induced rats. Meanwhile, kidney tissue from rats pre-treated with propolis before sepsis induction showed improvement, but with mild cognition and little interstitial hemorrhage of kidney tissue (Fig. 5).

\section{Immuno-histochemical analysis}

Similar to the control group, curcumin and propolis treatment showed weak expression of NF-kB in their kidney sections. Meanwhile, the kidney of septic rats showed a marked increase in NF-kB expression indicating its obvious activation. Curcumin pre-treatment inhibited and attenuated NF-kB activation in the kidney of septic rats as estimated by weak NF-kB expression in curumin- and septic-combined group, while propolis pre-treatment showed a moderate effect on its expression in kidney tissue of propolis- and sepsis-combined group (Fig. 6).

\section{Discussion}

Sepsis stimulates an immune response, causing activation of a cascade of immune activities resulting in tissue damage, multiple organ failure, and death. Sepsis pathology may be due to several factors, like bacterial or fungus infections. Septic acute kidney injury (S-AKI) is the most common cause of kidney injury in the ICU. In the previous study, Höcherl et al. [20] reported deterioration of kidney functions after induction of severe sepsis using LPS in vivo model. 


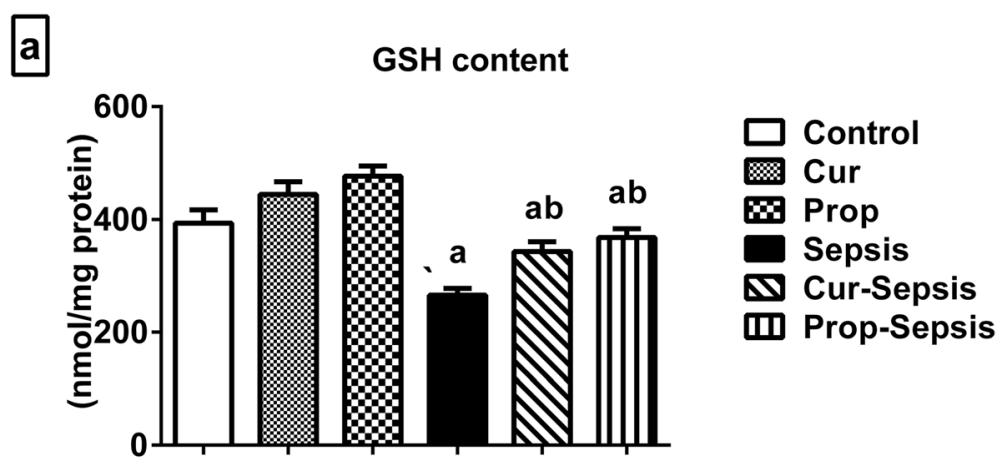

b

GPx activity

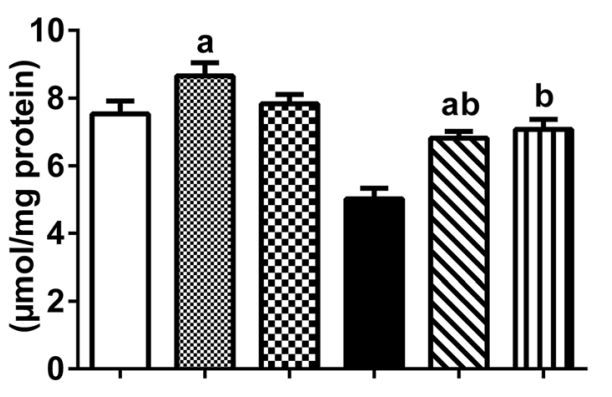

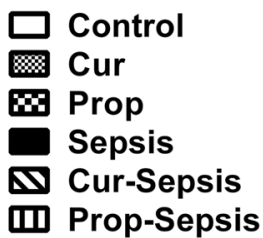

c

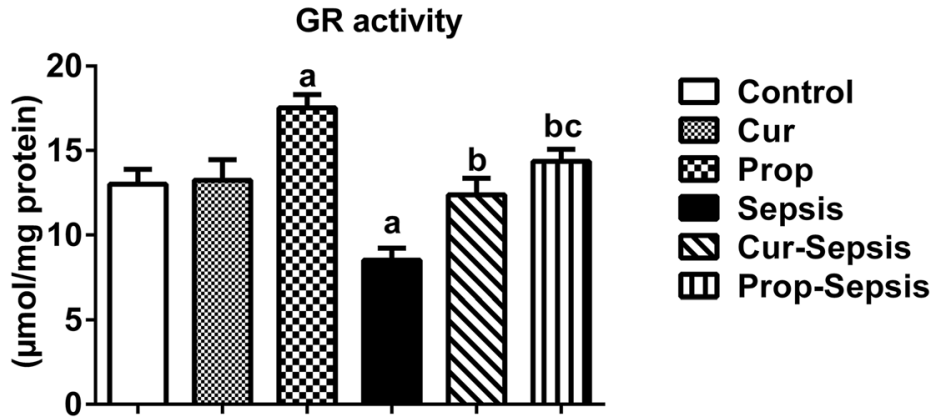

Fig. 1 The effect of either propolis (Prop) or curcumin (Cur) on a glutathione (GSH) content, b glutathione peroxidase (GPx), and c glutathione reductdase (GR) in kidney tissue homogenate after sepsis-induction in adult male rats. Data are expressed as mean \pm S.E.M for 6 rats/group. a, significant difference from control group by one-way ANOVA at $P<0.05$; b significant difference from SEP group by one-way ANOVA at $P<0.05$; c significant difference between cur and prop-sepsis and cur-sepsis groups by one-way ANOVA at $P<0.05$

In the present study, the cecal slurry injection increased oxidative stress markers in kidneys tissue homogenate. The reactive oxygen and nitrogen species and/ or free radicals may take apart in sepsis pathogenesis. Nitric oxide, superoxide, hydrogen peroxide, and hydroxyl radical may have a key role in endotoxemia. Nitric oxide is one of the vital parameter affecting sepsis. Cellular depletion of GSH can lead to apoptosis, presumably via activation of mitogen-activated protein kinase (MAPK) cascades in various cell models [21]. These results are in agreement with Andrades et al. [22] who reported that the oxidative stress caused proteins damage. The SOD and CAT found to be critical parameters in sepsis; elevation of SOD and CAT caused accumulation of $\mathrm{H}_{2} \mathrm{O}_{2}$ in cells. Meanwhile, Vasanthkumar et al. [23] reported similar increments in LPO and NO after sepsis induction in mice using LPS.

On the other hand, It is well established that proinflammatory cytokines, such as tumor necrosis factoralpha (TNF- $\alpha$ ), interleukin-1beta (IL-1 $\beta$ ), and IL-6, contribute to the development of AKI in septic patients [24]. Macrophages were documented to be the primary source of inflammatory (pro-inflammatory and antiinflammatory) mediators, thus playing a key role in modulating the host immune response [25]. IL-1 $\beta$ was found to be involved in the inflammatory symptoms 
a

LPO content

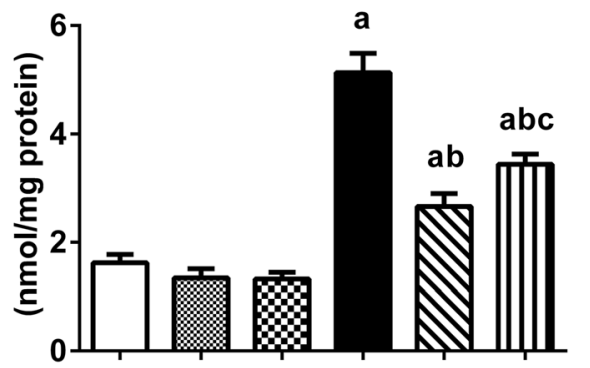

Control

Cur

Brop

Sepsis

$\nabla$ Cur-Sepsis

血 Prop-Sepsis

b

NO content

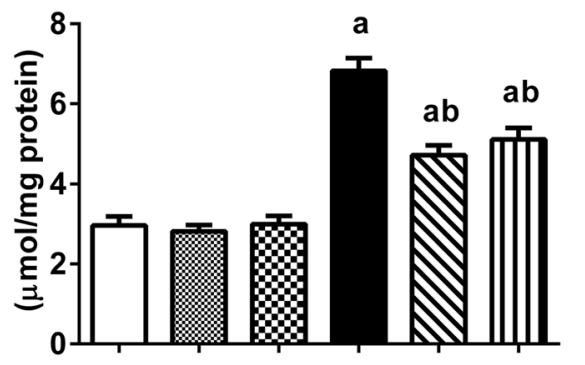

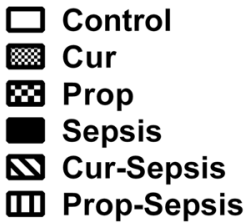

c

SOD activity

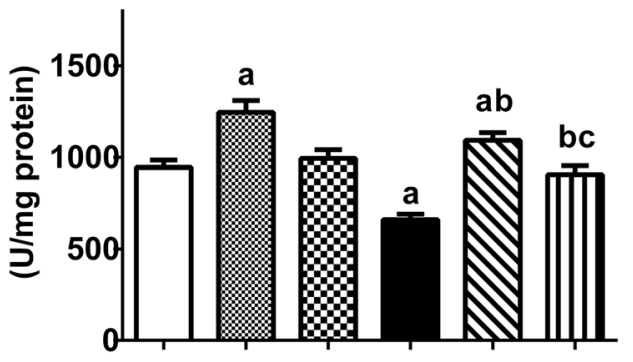

Control

\% Cur

W Prop

- Sepsis

ه Cur-Sepsis

II Prop-Sepsis

d

CAT activity
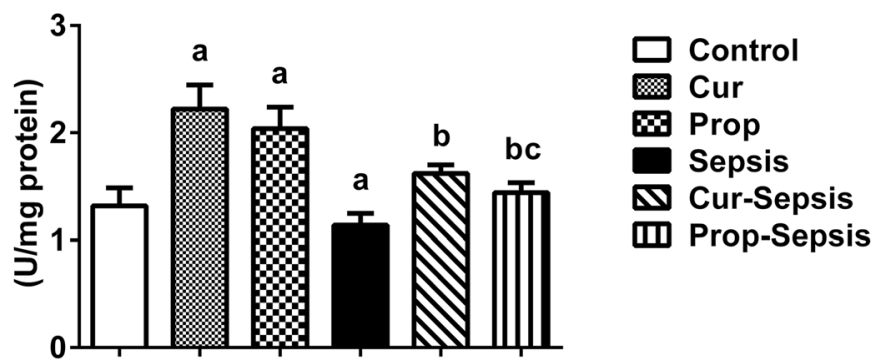

Fig. 2 The effect of either propolis (Prop) or curcumin (Cur) on a lipid peroxidation (LPO), b nitric oxide (NO), c superoxide dismutase (SOD), and $\mathbf{d}$ catalase (CAT) in kidney tissue homogenate after sepsis-induction in adult male rats. Data are expressed as mean \pm S.E.M for 6 rats/group. a significant difference from control group by one-way ANOVA at $P<0.05$; b significant difference from SEP group by one-way ANOVA at $P<0.05$; C, significant difference between cur and prop-sepsis and cur-sepsis groups by one-way ANOVA at $P<0.05$

(fever, lymphocyte responses, and neutrophil migration) and several inflammatory diseases. Besides, IL-1 $\beta$ was indicated to increase recruitment of inflammatory cells including the peritoneal cavit y[25].

Inflammatory cytokines produced by leukocytes activation such as TNF- $\alpha$, IL- $1 \alpha$, IL- $1 \beta$, and IL- 6 and chemokines such as IL-8, also contribute to sepsis severity. Reactive oxygen species activate the production of pro-inflammatory cytokines such as IL-1 $\beta$, IL-6, and TNF- $\alpha$. Furthermore, it triggered a downstream NF- $\kappa B$ activation, a pathway by which TNF- $\alpha$ induces apoptosis in a human monocytic cell line. 


\section{a}

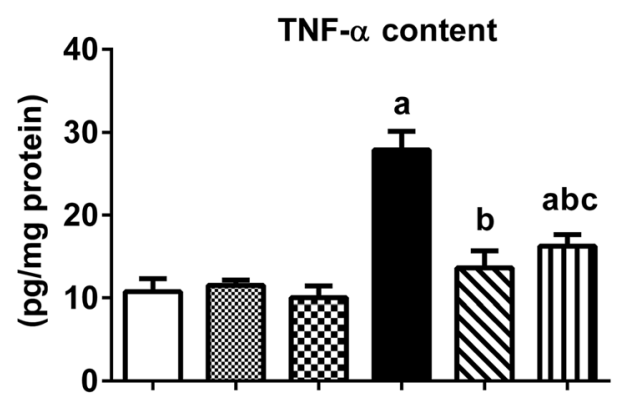

b

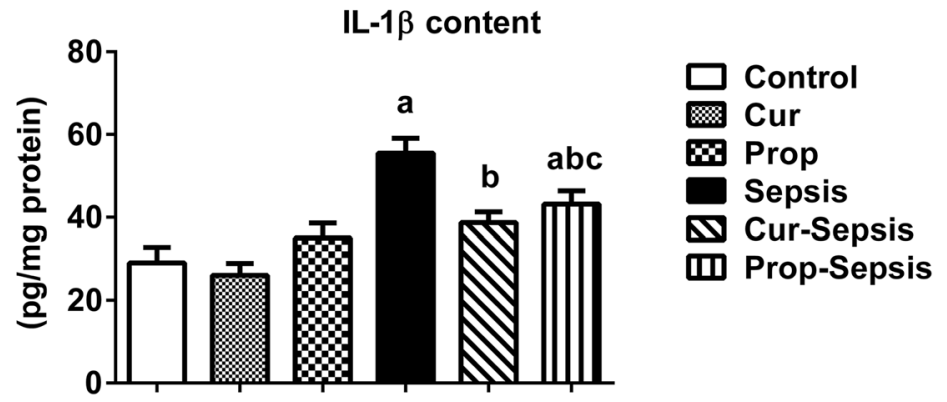

c

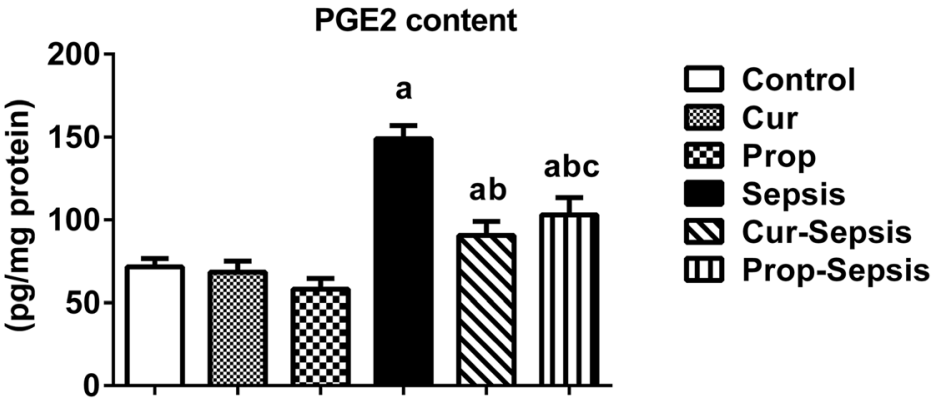

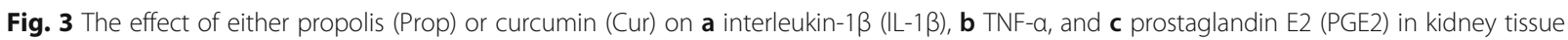
homogenate after sepsis-induction in adult male rats. Data are expressed as mean \pm S.E.M for 6 rats/group. a, significant difference from control group by one-way ANOVA at $P<0.05$; b significant difference from SEP group a by one-way ANOVA at $P<0.05$; $c$, significant difference between cur and prop-sepsis and cur-sepsis groups by one-way ANOVA at $P<0.05$

Moreover, the over release of $\mathrm{NO}$ due to inducible iNOS activity has been accompanied by general vasodilatation and consequently hypotension. Expression of iNOS is usually controlled by NF- $\mathrm{kB}$ activation in sepsis. The overproduction of $\mathrm{NO}$ was found to mediate apoptosis in some diseases including septic shock through the endoplasmic reticulum stress pathway in some cell types as it reacts with proteins and nucleic acids causing cytotoxic effects elicited by DNA or mitochondrial damage [26]. Increased $\mathrm{NO}$ and PGs release, vasodilator mediators, and the downregulation of vasoconstrictive receptors were among several mechanisms implicated in the failure of vascular smooth muscle cells proper constriction properly and the mediation of endotoxemia- related vasodilation [27]. Hemodynamics as well as inflammatory changes were suggested to be contributed in the development of renal impairment [28]. The present results showed a marked elevation in inflammatory markers in kidney tissues of sepsis group as well as marked rise in Bax content with a significant depletion in $\mathrm{Bcl}-2$ content that are the key proteins critical for apoptosis.

Pathogenic bacteria and their products trigger the activation of NF- $\mathrm{kB}$, playing a central role in the formation of networks between cytokines and the inflammatory mediators, leading to the pathophysiology of septic shock [5, 29]. The inflammatory process activates a series of receptors and transcription factors such as NF- 


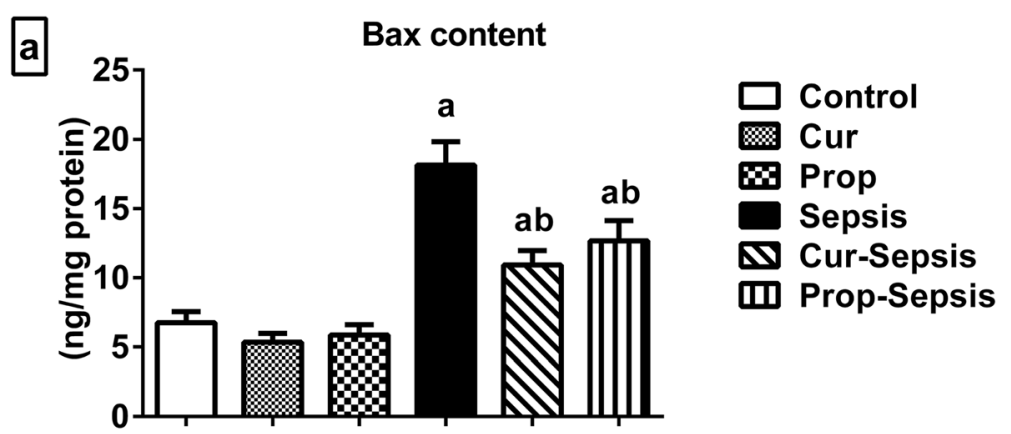

b Bcl-2 content
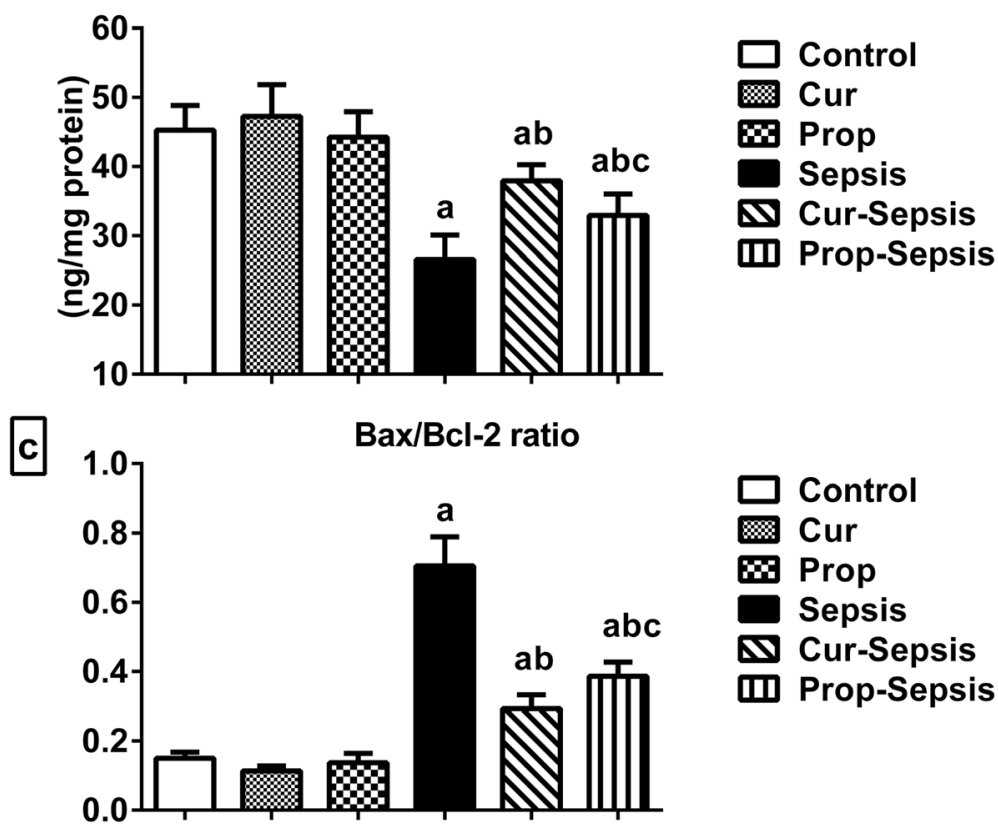

Fig. 4 The effect of either propolis (Prop) or curcumin (Cur) on a Bax, b BcL-2 contents, and $\mathbf{c}$ the Bax/BcL-2 ratio in kidney tissue after sepsisinduction in adult male rats. Data are expressed as mean \pm S.E.M for 6 rats/group. a, significant difference from control group by one way ANOVA at $P<0.05 ; \mathrm{b}$, significant difference from SEP group by one-way ANOVA at $P<0.05 ; \mathrm{c}$, significant difference between cur and prop-sepsis and cur-sepsis groups by one-way ANOVA at $P<0.05$

$\mathrm{\kappa B}$ and the receptor for advanced glycation products, which lead to $\beta$ cell dysfunction and apoptosis.

PGEs are important paracrine regulators of kidney function produced from arachidonic acid and exert their action via the PGE receptors [30]. PEGs affect renal vascular resistance, glomerular filtration rate, tubular reabsorption of salt and water, and renin secretion as indicated by Hao and Breyer [31]. The present study indicated increased renal contents of PGE2 in kidney homogenate of sepsis-induced rats, agreeing with previous studies of Höcherl et al. [32] which indicated huge increments in PGE2 in response to LPS treatment.

Recently, Meurer et al. [33] found increased adrenocortical and medullary tissue contents of PGE2 in response to endotoxemia-related AKI. PEG2 was found to exert a dual effect on renal vascular tone, inducing vasodilatation at lower concentrations and vasoconstriction at higher concentrations. The stimulated vasodilator PGE2 and PGI2 system might be regarded beneficial in sepsis, only within the kidney, thus protecting the kidney against endotoxemia-related injury.

Several of antioxidant and anti-inflammatory markers were performed in the present study to distinguish the potency of both curcumin and propolis on the kidney disorder in the endotoxemic rat. Though both curcumin and propolis, in the present study, were found to modulate the oxidative stress as well as inflammatory markers in kidney tissue of sepsis-induced rats, the therapeutic potential of curcumin is highly considered due to its ability to inhibit NF-kB activation and its downstream 


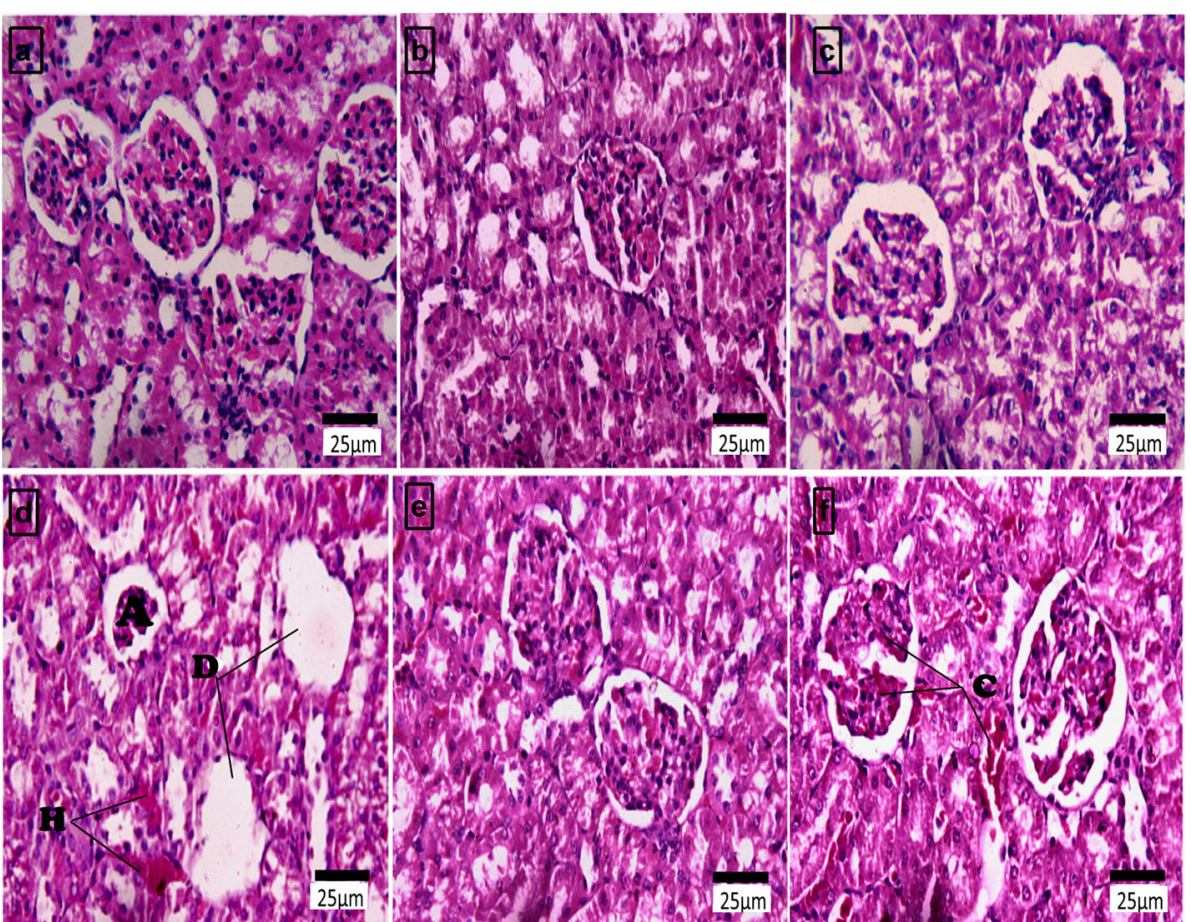

Fig. 5 A photomicrograph of a section of kidney tissue (a-f). a The normal structure of kidney. Notice normal glomeruli with an intact Bowman's capsule and proximal convoluted capsule $(\times 400, \mathrm{H} \& \mathrm{E})$. b A photomicrograph of another section of kidney tissue for rat treated with curcumin shows the normal structure of this tissue $(\times 400, \mathrm{H} \& \mathrm{E})$. c A photomicrograph of a section of liver tissue from a rat received propolis shows a quite normal structure of kidney tissue ( $\times 400$, H\&E). $\mathbf{d}$ A photomicrograph of a section of kidney tissue of sepsis-induced rat shows dilation of interstitial tubule (D) severe tubular leakage appeared, atrophic glomeruli (A), and interstitial hemorrhages $(H)(\times 400, H \& E)$. e A photomicrograph of a section of kidney tissue from a rat subjected to sepsis after treated with curcumin showed an improvement ( $\times 400, \mathrm{H} \&$ E). $\mathbf{f}$ A photomicrograph of a section of kidney tissue from a rat received propolis before sepsis induction showed improvement, but mild cognition $(C)$ and mild interstitial hemorrhages $(H)$ of kidney tissue still found $(\times 400, \mathrm{H} \& \mathrm{E})$

genes including IL-1 $\beta$, TNF- $\alpha$, and IL-6 expression [34]. As NF-kB regulates the expression of over 500 genes associated with inflammation, tumorigenesis, cellular sur$\mathrm{vival} /$ proliferation, and chemoresistance [35].

Curcumin inhibited NF- $\mathrm{kB}$ activation in sepsisinduced muscle protein degradation by preventing the phosphorylation and degradation of IkB $\alpha$ thus resulting in inhibition of inflammation and exert other antiinflammatory effects, including oxygen radical scavenging [36].

The desirable protective or putative therapeutic properties of curcumin may be regarding its antioxidant and anti-inflammatory properties. Yang et al. [37] reported a reduction in proteinuria and inflammatory markers in diabetic nephropathy treated with curcumin.

Propolis has wide biological and potential therapeutic impacts due to anti-inflammatory and the antioxidant action of CAPE (the major component of propolis), regarding its ability to inhibit the systemic inflammatory response, NF- $\mathrm{kB}$, and apoptosis.

Teles et al. [38] reported the administration of red propolis to $5 / 6$ renal ablation animal model was found to partially reduce kidney hypertension, proteinuria, and serum creatinine, infiltration, and reduced oxidative stress. He attributed its renoprotection property to reduction of both renal inflammation and oxidative stress.

Pinocembrin, is the phenolic compound found in propolis, reduces the content of proinflammatory cytokines (TNF- $\alpha$, interleukin-1beta (IL-1 $\beta)$ ), chemokines, inducible nitric oxide synthase (iNOS), and aquaporin-4 [39]. Another study suggested that pinocembrin appears to suppress the nuclear translocation of NF- $\mathrm{KB}$ and decrease TNF- $\alpha$ expression [40].

Curcumin treatment was reported to increase GSH content and the activity of antioxidant enzymes as documented by Mylonas and Kouretas [41]. In vivo study by Qiu et al. [42] demonstrated curability to increase GSH content and to upregulate SOD, CAT activities in the liver of a murine model after glycerol-induction of nephrotoxicity. Curcumin markedly inhibited hemolysis and lipid peroxidation of erythrocytes induced by linoleate by functioning as a scavenger of $\mathrm{NO}$ and blocking its synthesizing enzyme [25]. Curcumin also markedly decreased LPO and NO in the kidney of sepsis-induced 


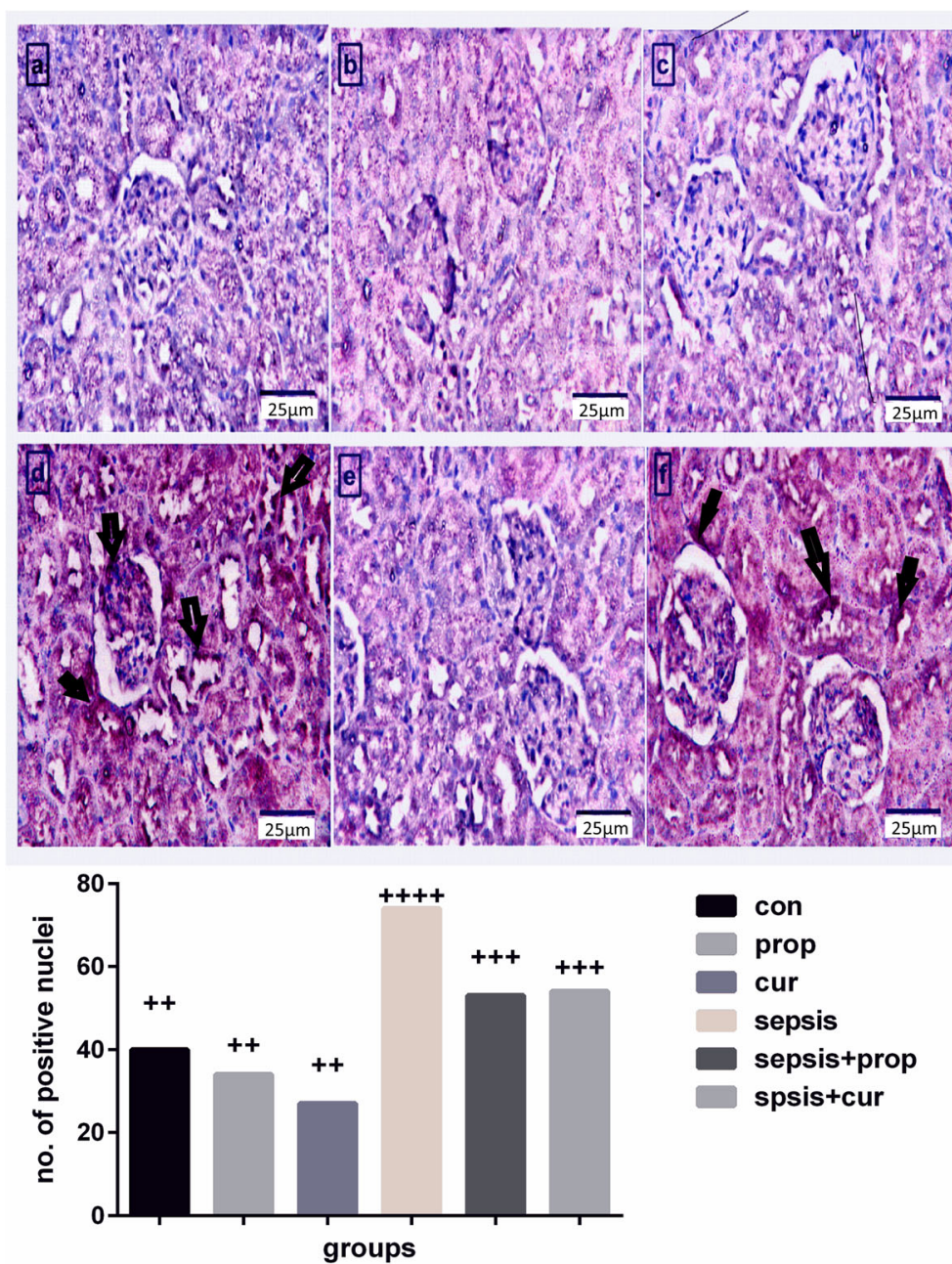

Fig. 6 Immunohistochemical expression of NF-kB in rat kidney sections with magnification $\times 400$. A photographic of kidney section of rat were treated with vehicle showing weak expression of NF-kB (a), another section of kidney tissue for rat treated with curcumin showing weak expression of NF-kB (b), kidney section of rat treated with propolis showing weak expression of NF-kB (c), kidney section of rat after induction of sepsis showed strong expression of NF-kB (d), a section of kidney tissue from a rat subjected to sepsis after treated with curcumin showing weak expression of NF-kB (e), a section of kidney tissue from a rat subjected to sepsis after treated with propolis showing moderate expression of NFkB (f). Arrows show immunopositive nuclei

mice [23]. C66, a novel curcumin derivative, was reported to reduce TNF- $\alpha$, IL- $1 \beta, \mathrm{COX}-2$, and NF- $\mathrm{kB}$ production, in high glucose-stimulated diabetic rats [43].

On the other hand, Sabuncuoglu et al. [11] reported that propolis providing an alternative therapy against resistant strains infections by elevation GSH content and reduced bacterial translocation in the ileum after bile duct ligation by enhancing mucosal barrier function, thus reducing bacterial overgrowth.

Malondialdehyde concentrations are commonly being used as potential oxidative stress biomarkers and indicators of oxidative lipid damage. The chemical structure of the constituent polyphenols enables propolis to eliminate free radicals. The flavonoids in propolis are powerful antioxidants capable of scavenging free radicals and thereby protecting the cell membrane against lipid peroxidation [44]

In the present study, curcumin reduced the production of pro-inflammatory mediators in the sepsis rat model. This reduction could be due to inhibited cyclic AMP signaling in macrophages. Under normal conditions, NF- $\mathrm{B}$ is bound to be an inhibitor and sequester in the cytoplasm [45]. The regulatory pathway triggers the inflammatory processes, which in turn regulate the inflammatory process via activation of the inflammatory cytokines; PGE2 and NO. Moreover, Wang et al. [45] indicated translocation of NF-kB, after LPS treatment, from the cytoplasm to the nucleus initiating the release of various inflammatory mediators: iNOS, COX-2, NO, and PGE2. 
The anti-inflammatory activity of propolis appears related to its associated constituents: flavonoids, phenolic acids and their esters, terpenoids, steroids, and amino acids, with CAPE being the most studied compound. The main mechanisms underlying the antiinflammatory activity of propolis include (1) the inhibition of cyclooxygenase (COX) and consequent inhibition of prostaglandin biosynthesis, (2) free radical scavenging as discussed below; (3) inhibition of nitric oxide synthesis; (4) reduction in the concentration of inflammatory cytokines; and (5) immunosuppressive activity [46].

Bcl-2 (a member of proteins family) maintain cellular homeostasis and regular apoptosis by various cytokines including IL- $1 \beta$, so $\mathrm{Bcl}-2$ has very critical role in many biological processes and diseases [37]. Bcl-2 family members were considered as key regulators that control the release of cytochrome $C$ and other apoptosis-promoting factors from mitochondria. Therefore, the present work suggests that overexpression of Bcl-2 prevented apoptosis in the kidney of curcumin pre-treated septic rats. In the present study, curcumin pre-treatment markedly inhibited Bax content parallel to elevated $\mathrm{Bcl}-2$ contents in kidney tissues more than the propolis did. Apoptosis could be mediated by NO through the endoplasmic reticulum and the transcriptional factors (stress pathway), taking place by Bax acting as proapoptotic molecules located in the cytosol under non-apoptotic conditions and translocated to the mitochondria in response to apoptotic stimuli. NO-induced apoptosis through a mechanism involving cytochrome $\mathrm{C}$ release from mitochondria [47]. Scorrano et al. [48] reported that Bax and Bak were to operate on endoplasmic reticulum as well as mitochondria in maintaining $\mathrm{Ca} 2+$ homeostasis.

In addition, histopathological examination revealed that cecal slurry injection caused acute kidney injury represented by capillary congestion, leukocyte infiltration, tubular degeneration, and the formation of casts and luminal debris due to oxidative stress that caused proinflammatory cytokines storm and apoptosis. These results are in agreement with Liu et al. [49] and Wang et al. [50]. Kidneys of septic rats pretreated with curcumin indicated an improvement compared with a kidney of sepsis-induced rats due to the protective effect of curcumin against oxidative stress and inhibition of cytokines release as previously reported by Yang et al. [37] and Moneim et al. [10]. Meanwhile, kidney tissue from rats pre-treated with propolis before sepsis induction showed protective impact against toxic effect of sepsis; these results are in agreement with Sameni et al. [51] although mild cognition and little interstitial hemorrhage of kidney tissue is still seen.

\section{Conclusion}

The present study indicates that both curcumin and propolis protect rats' kidneys after sepsis induction. Propolis is partially diminishing sepsis consequent to renal injury, and curcumin have additional protective role via inhibition of the IL-1 $\beta$ that is responsible for production of NO and PGE2 in sepsis-induced renal injured rats. Curcumin also has an anti-inflammatory effect in preventing NO-mediated apoptosis upstream and downregulation of NF- $\mathrm{kB}$ activity, which is the clue preventive mechanism of renal cell apoptosis. In conclusion, both propolis and curcumin exhibited renoprotective effects as they have antioxidant, anti-inflammatory properties, especially curcumin that exerted the most pronounced effect as an anti-inflammatory and antiapoptotic reflecting its powerful effect against bacteria and sepsis-induced kidney injury.

\section{Abbreviations \\ AKI: Acute kidney injury; BAX: BCl-2-associated X protein; $\mathrm{BCl}-2$ : B cell lymphoma 2; Ca+: Calcium ion; CAT: Catalase; Cox: Cyclooxygenase; Cur: Curcumin; GPx: Glutathione peroxidase; GSH: Reduced glutathione; GSH- Px: Glutathione peroxidase; GSR or GR: Glutathione reductase; \\ GSSG: Glutathione disulfide; IL: Interleukin; MAPK: Mitogen-activated protein kinase; MDA: Malondialdhyde; NO: Nitric oxide; NF-kB: Inhibitor of Nuclear Factor kappa-B; PGE2: Prostaglandin E2; PI3K: Phosphatidylinositol 3-kinase; PKB: Protein kinase B; PROP: Propolis; ROS: Reactive oxygen species; \\ Seps: Sepsis; SOD: Superoxide dismutase; TNF-a: Tumor necrosis factor alpha}

\section{Acknowledgements}

The authors are so grateful to the head and members of physiology department and to the animal house of NODCAR.

\section{Plant source}

Curcumin powder (curcuminoid 95.02\%) was obtained from Sigma chemical company (Sigma-Aldrich, USA).

Propolis was purchased from Agriculture College, Cairo University.

\section{Studies involving plants}

As per the local and national guidelines and legislation and the required or appropriate permissions and/or licenses for the study.

\section{Authors' contributions}

AMA and SMM designed the study. AMA and SMM collected the blood and tissue samples. AEA, SMM, and AMA performed biochemical examination and interpreted the histopathological and immunohistochemical studies. SMM wrote the paper, and AMA wrote the final form. All authors revised, edited the paper, and accepted it for publication.

\section{Funding}

This research did not receive any specific grant from any funding agency.

Availability of data and materials

Data and materials are available upon request.

\section{Ethics approval and consent to participate}

All experimental protocols and steps of the tests were conducted in compliance with the regulations of the Research Ethics Committee of Egyptian Ethical Guidelines for the use of animals in research. Additionally, all animal experiments were obtained from animal house of NODCAR, in accordance with protocols approved by the United States National Institutes of Health (NIH, 1978). This study was also approved and supported by Ethics Committee of NODCAR (http://www.nodcar.eg.net/) No. (NODCAR/II/21/19).

Consent for publication

Non-applicable. 


\section{Competing interests}

The authors declare that they have no conflict of interest.

\section{Author details}

'Researcher at Department of Physiology, National Organization of Drug Control, and Research, Cairo, Egypt. ${ }^{2}$ Department of Zoology, Faculty of Science, Cairo University, Cairo, Egypt. ${ }^{3}$ Department of Zoology \& Entomology Faculty of Science, Helwan University, Helwan, Egypt.

Received: 1 June 2020 Accepted: 2 September 2020

\section{Published online: 08 November 2020}

\section{References}

1. Hotchkiss RS, Nicholson DW (2006) Apoptosis and caspases regulate death and inflammation in sepsis. Nat Rev Immunol 6:813-822. https://doi.org/10. 1038/nri1943

2. Singer M, Deutschman CS, Seymour CW, Shankar-Hari M, Annane D, Bauer M, Bellomo R, Bernard GR, Chiche J-D, Coopersmith CM, Hotchkiss RS, Levy MM, Marshall JC, Martin GS, Opal SM, Rubenfeld GD, van der Poll T, Vincent J-L, Angus DC (2016) The Third International Consensus Definitions for Sepsis and Septic Shock (Sepsis-3). JAMA 315:801. https://doi.org/10.1001/ jama.2016.0287

3. Messaris E, Memos N, Chatzigianni E, Kataki A, Nikolopoulou M, Manouras A, Albanopoulos K, Konstadoulakis MM, Bramis J (2008) Apoptotic death of renal tubular cells in experimental sepsis. Surg Infect 9:377-388. https://doi. org/10.1089/sur.2006.018

4. Lei L, Wang M-J, Zhang S, Hu D-J (2020) Effects of prostaglandin E combined with continuous renal replacement therapy on septic acute kidney injury. World J Clin Cases 8:2738-2748. https://doi.org/10.12998/wjcc. V8.113.2738

5. Liu SF, Malik AB (2006) NF-KB activation as a pathological mechanism of septic shock and inflammation. Am J Physiol Cell Mol Physiol 290:L622L645. https://doi.org/10.1152/ajplung.00477.2005

6. Goel A, Kunnumakkara AB, Aggarwal BB (2008) Curcumin as "curecumin": from kitchen to clinic. Biochem Pharmacol 75:787-809. https://doi.org/10. 1016/.j.bcp.2007.08.016

7. Lewis AJ, Seymour CW, Rosengart MR (2016) Current Murine Models of Sepsis. Surg Infect 17:385-393. https://doi.org/10.1089/sur.2016.021

8. Solov'eva T, Davydova V, Krasikova I, Yermak I (2013) Marine compounds with therapeutic potential in gram-negative sepsis. Mar Drugs 11:2216-2229

9. El-Nabarawy SK, Radwan OK, El-Sisi SF, Abdel-Razek AM (2015) Comparative Study of Some Natural and Artificial Food Coloring Agents on Depression, Anxiety and Anti-Social Behavior in Weanling Rats. IOSR J Pharm Biol Sci Ver III 10:2319-7676. https://doi.org/10.9790/3008-10238389

10. Moneim AEA, Mahmoud SM, Abd-Elrazek AM, Sisi SFE, Mohammed IS, ElYamany NA (2019) Curcumin alleviates liver injury and altered monoamines content in cerebral cortex of sepsis-induced rats. J Innov Pharm Biol Sci 6: $11-23$

11. Sabuncuoglu MZ, Kismet K, Kilicoglu SS, Kilicoglu B, Erel S, Muratoglu S, Sunay AE, Erdemli E, Akkus MA (2007) Propolis reduces bacterial translocation and intestinal villus atrophy in experimental obstructive jaundice. World J Gastroenterol 13:5226. https://doi.org/10.3748/WJG.V13.139. 5226

12. Abd-Elrazek AM, El-dash HA, Said NI (2020) The role of propolis against paclitaxel-induced oligospermia, sperm abnormality, oxidative stress and DNA damage in testes of male rats. Andrologia 52:1-10. https://doi.org/10. 1111/and.13394

13. Esmat A, Mahmoud S, Mohamed A, Fahmy E, Salah M, Ahmed E. (2019). Propolis Extract Attenuates Sepsis-Induced Hepatotoxicity and Neurotoxicity in Male Rats. Egyptian Academic Journal of Biological Sciences. C, Physiology and Molecular Biology, 11(3), 1-19. https://doi.org/10.21608/ eajbsc.2019.44505

14. Da Costa MFB, Libório AB, Teles F, Da Silva MC, Soares PMG, Meneses GC, De Paulo Rodrigues FA, Leal LKAM, Miron D, Silva AH, Martins AMC (2015) Red propolis ameliorates ischemic-reperfusion acute kidney injury. Phytomedicine 22:787-795. https://doi.org/10.1016/.jphymed.2015.03.017

15. Chen HW, Kuo HT, Chai CY, Ou JL, Yang RC (2007) Pretreatment of curcumin attenuates coagulopathy and renal injury in LPS-induced endotoxemia. J Endotoxin Res 13:15-23. https://doi.org/10.1177/ 0968051907078605
16. Lee MJ, Kim K, Jo YH, Lee JH, Hwang JE (2016) Dose-dependent mortality and organ injury in a cecal slurry peritonitis model. J Surg Res 206:427-434. https://doi.org/10.1016/j.jss.2016.08.054

17. El Menyiy N, Al Waili N, Bakour M, Al-Waili H, Lyoussi B (2016) Protective Effect of Propolis in Proteinuria, Crystaluria, Nephrotoxicity and Hepatotoxicity Induced by Ethylene Glycol Ingestion. Arch Med Res 47:526534. https://doi.org/10.1016/j.arcmed.2016.12.010

18. Bancroft JD, Cook HC (1994) Manual of histological techniques and their diagnostic application. 457

19. Arabaci T, Cicek Y, Canakci V, Canakci CF, Ozgoz M, Albayrak M, Keles ON (2010) Immunohistochemical and stereologic analysis of NF-KB activation in chronic periodontitis. Eur J Dent 4:454-461

20. Höcherl K, Schmidt C, Kurt B, Bucher M (2008) Activation of the PGI $2 / / P$ system contributes to the development of circulatory failure in a rat model of endotoxic shock. Hypertension 52:330-335. https://doi.org/10.1161/ HYPERTENSIONAHA.108.112029

21. Lu GD, Shen H-M, Chung MCM, Ong CN (2007) Critical role of oxidative stress and sustained JNK activation in aloe-emodin-mediated apoptotic cell death in human hepatoma cells. Carcinogenesis 28:1937-1945. https://doi. org/10.1093/carcin/bgm143

22. Andrades M, Ritter C, Moreira JCF, Dal-Pizzol F (2005) Oxidative parameters differences during non-lethal and lethal sepsis development1. J Surg Res 125:68-72. https://doi.org/10.1016/J.JSS.2004.11.008

23. Vasanthkumar T, Manjunatha Hanumanthappa PB (2018) Protective effect of dietary curcumin and capsaicin on LPS-induced inflammation in mice. Pharm J 4:725-729. https://doi.org/10.5530/pj.2018.4.121

24. LV J, Wu ZL, Gan Z, Gui P, Yao SL (2020) CXCL14 overexpression attenuates sepsis-associated acute kidney injury by inhibiting proinflammatory cytokine production. Mediators Inflamm 2020: https://doi.org/10.1155/2020/2431705

25. Schneider CP, Schwacha MG, Chaudry IH (2004) The role of interleukin-10 in the regulation of the systemic inflammatory response following traumahemorrhage. Biochim Biophys Acta Mol basis Dis 1689:22-32. https://doi. org/10.1016/j.bbadis.2004.01.003

26. Kawahara K, Oyadomari S, Gotoh T, Kohsaka S, Nakayama H, Mori M (2001) Induction of CHOP and apoptosis by nitric oxide in p53-deficient microglial cells. FEBS Lett 506:135-139 . https://doi.org/10.1016/50014-5793(01)02898-8

27. Levy B, Fritz C, Tahon E, Jacquot A, Auchet T, Kimmoun A (2018) Vasoplegia treatments: the past, the present, and the future. Crit Care 22:52. https://doi. org/10.1186/s13054-018-1967-3

28. Lipcsey M, Bellomo R (2011) Septic acute kidney injury: hemodynamic syndrome, inflammatory disorder, or both? Crit Care 15:1008. https://doi. org/10.1186/cc10525

29. Mahmoud SM, El-Yamany NA (2012) The protective effect of propolis on norepinephrine, dopamine and 5-hydroxytryptamine content in thalamushypothalamus and cerebellum of endotoxin-intoxicated adult male albino rats. Life Sci J 9:3372-3379

30. Watanabe Y, Namba A, Honda K, Aida Y, Matsumura H, Shimizu O, Suzuki N, Tanabe N, Maeno M (2009) IL-1 $\beta$ stimulates the expression of prostaglandin receptor ep4 in human chondrocytes by increasing production of prostaglandin E 2. Connect Tissue Res 50:186-193. https://doi.org/10.1080/ 03008200802588451

31. Hao C-M, Breyer MD (2008) Physiological regulation of prostaglandins in the kidney. Annu Rev Physiol 70:357-377. https://doi.org/10.1146/annurev. physiol.70.113006.100614

32. Höcherl K, Dreher F, Kurtz A, Bucher M (2002) Cyclooxygenase-2 inhibition attenuates lipopolysaccharide-induced cardiovascular failure. Hypertens (Dallas, Tex 1979) 40:947-953

33. Meurer M, Ebert K, Schweda F, Höcherl K (2018) The renal vasodilatory effect of prostaglandins is ameliorated in isolated-perfused kidneys of endotoxemic mice. Pflugers Arch - Eur J Physiol 470:1691-1703. https://doi. org/10.1007/s00424-018-2183-3

34. Sandur SK, Pandey MK, Sung B, Ahn KS, Murakami A, Sethi G, Limtrakul P, Badmaev V, Aggarwal BB (2007) Curcumin, demethoxycurcumin, bisdemethoxycurcumin, tetrahydrocurcumin and turmerones differentially regulate anti-inflammatory and anti-proliferative responses through a ROSindependent mechanism. Carcinogenesis 28:1765-1773. https://doi.org/10. 1093/carcin/bgm123

35. Min S-W, Jung S-H, Cho K-H, Kim D-H (2008) Antihyperlipidemic effects of red ginseng, Crataegii Fructus and their main constituents ginsenoside Rg3 and ursolic acid in mice. Biomol Ther 16:364-369. https://doi.org/10.4062/ biomolther.2008.16.4.364 
36. Asai A, Miyazawa T (2001) Dietary curcuminoids prevent high-fat dietinduced lipid accumulation in rat liver and epididymal adipose tissue. J Nutr 131:2932-2935

37. Yang H, Xu W, Zhou Z, Liu J, Li X, Chen L, Weng J, Yu Z (2015) Curcumin attenuates urinary excretion of albumin in type II diabetic patients with enhancing nuclear factor erythroid-derived 2-like 2 (Nrf2) system and repressing inflammatory signaling efficacies. Exp Clin Endocrinol Diabetes 123:360-367. https://doi.org/10.1055/s-0035-1545345

38. Teles F, Machado FG, Ventura BH, Malheiros DMAC, Fujihara CK, Silva LFF, Zatz $R$ (2009) Regression of glomerular injury by losartan in experimental diabetic nephropathy. Kidney Int 75:72-79. https://doi.org/10.1038/ki.2008.528

39. Lan X, Wang W, Li Q, Wang J (2016) The natural flavonoid pinocembrin: molecular targets and potential therapeutic applications. Mol Neurobiol 53: 1794-1801

40. Pei B, Sun J (2018) Pinocembrin alleviates cognition deficits by inhibiting inflammation in diabetic mice. J Neuroimmunol 314:42-49. https://doi.org/ 10.1016/.jneuroim.2017.11.006

41. Mylonas C, Kouretas D. Lipid peroxidation and tissue damage. In Vivo. 1999; 13(3):295-309.

42. Qiu P, Sun J, Man S, Yang H, Ma L, Yu P, Gao W (2017) Curcumin attenuates $N$-nitrosodiethylamine-induced liver injury in mice by utilizing the method of metabonomics. J Agric Food Chem 65:2000-2007. https://doi.org/10. 1021/acs.jafc.6b04797

43. Pan $H$, Wang $H$, Wang $X$, Zhu L, Mao L (2012) The absence of Nrf2 enhances NF-KB-dependent inflammation following scratch injury in mouse primary cultured astrocytes. Mediat Inflamm 2012:217580. https://doi.org/10 1155/2012/217580

44. Braakhuis A (2019) Evidence on the health benefits of supplemental propolis. Nutrients 11. https://doi.org/10.3390/nu11112705

45. Wang L, Pan H-T, Wang B, Liu Y, Fei K, Han W-F (2016) Allicin reduces IL-1ßinduced inflammatory cytokines via attenuating the NF-KB and MMP3 activation in human osteoarthritis chondrocytes model

46. Araujo MAR, Libério SA, Guerra RNM, Ribeiro MNS, Nascimento FRF (2011) Mechanisms of action underlying the anti-inflammatory and immunomodulatory effects of propolis: A brief review. Brazilian J Pharmacogn 22:208-219

47. Gotoh T, Terada K. Mori M (2001) hsp70-DnaJ chaperone pairs prevent nitric oxide-mediated apoptosis in RAW 264.7 macrophages. Cell Death Differ 8: 357-366. https://doi.org/10.1038/sj.cdd. 4400829

48. Scorrano L, Oakes SA, Opferman JT, Cheng EH, Sorcinelli MD, Pozzan T, Korsmeyer SJ (2003) BAX and BAK regulation of endoplasmic reticulum Ca2+: a control point for apoptosis. Science (80- ) 300:135-139. https://doi. org/10.1126/science.1081208

49. Liu C, Hu J, Mao Z, Kang H, Liu H, Fu W, Lv Y, Zhou F (2017) Acute kidney injury and inflammatory response of sepsis following cecal ligation and puncture in d-galactose-induced aging rats. Clin Interv Aging 12:593. https://doi.org/10.2147/CIA.S132277

50. Wang K, Xie S, Xiao K, Yan P, He W, Xie L (2018) Biomarkers of sepsisinduced acute kidney injury. Biomed Res Int 2018: https://doi.org/10.1155/ 2018/6937947

51. Sameni HR, Ramhormozi P, Bandegi AR, Taherian AA, Mirmohammadkhani M, Safari M (2016) Effects of ethanol extract of propolis on histopathological changes and anti-oxidant defense of kidney in a rat model for type 1 diabetes mellitus. J Diabetes Investig 7:506-513. https://doi.org/10.1111/jdi. 12459

\section{Publisher's Note}

Springer Nature remains neutral with regard to jurisdictional claims in published maps and institutional affiliations.

\section{Submit your manuscript to a SpringerOpen ${ }^{\circ}$ journal and benefit from:}

- Convenient online submission

- Rigorous peer review

- Open access: articles freely available online

- High visibility within the field

Retaining the copyright to your article

Submit your next manuscript at $\boldsymbol{\nabla}$ springeropen.com 